
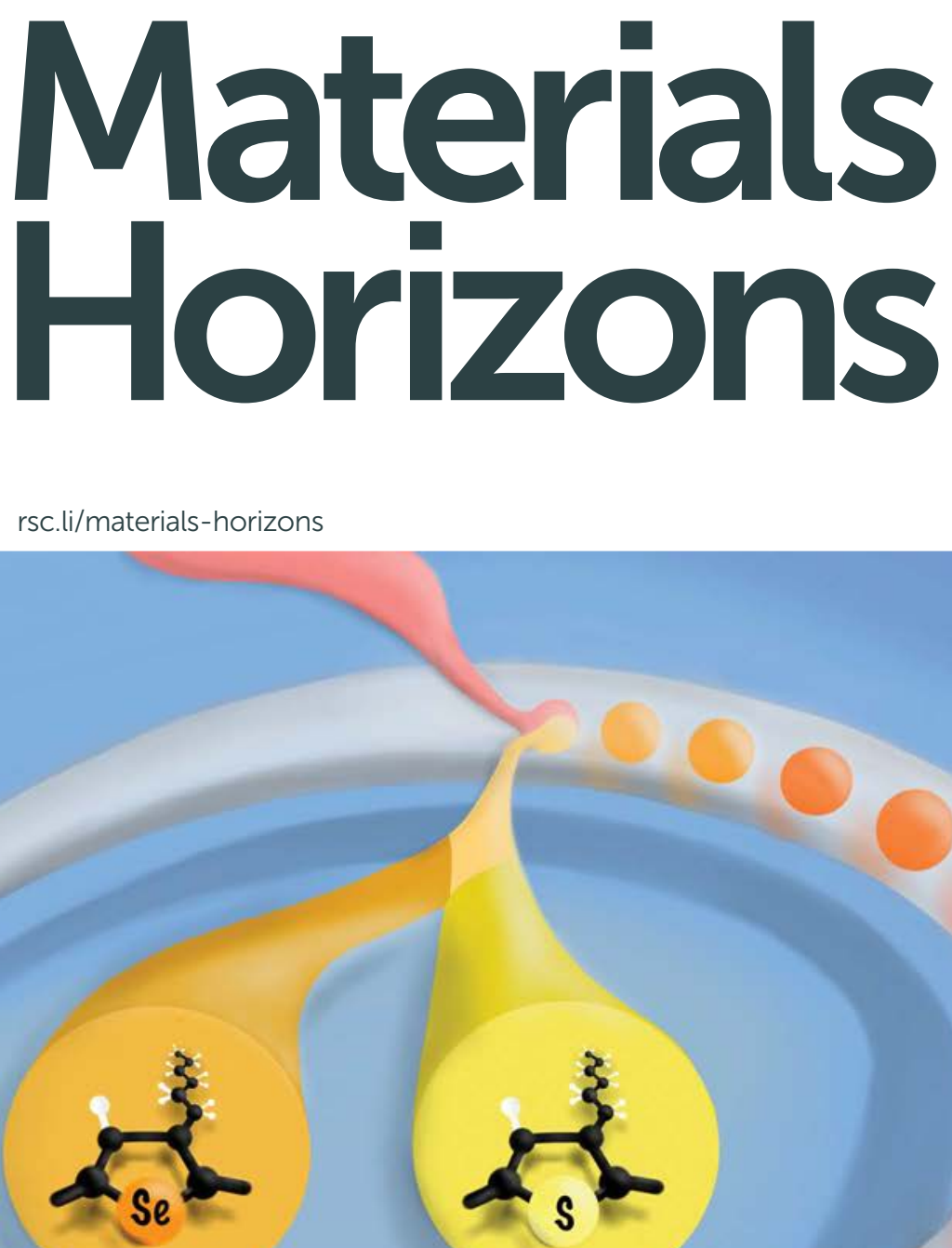

ISSN 2051-6347
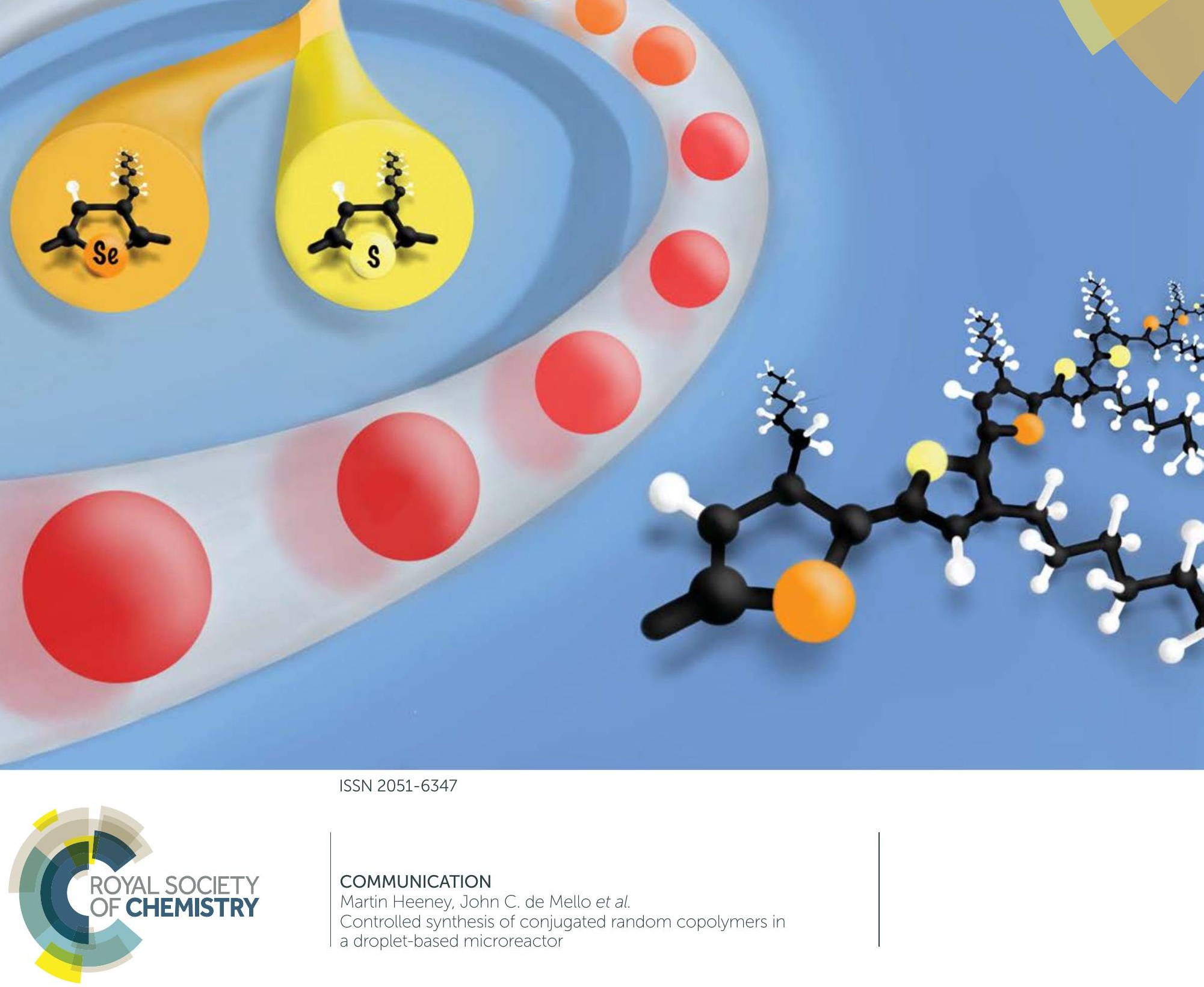


\title{
Controlled synthesis of conjugated random copolymers in a droplet-based microreactor
}

Cite this: Mater. Horiz., 2014, 1, 214

\author{
James H. Bannock, ${ }^{a}$ Mohammed Al-Hashimi, ${ }^{\text {ab }}$ Siva H. Krishnadasan, ${ }^{\text {ac }}$
}

Received 25th July 2013 Jonathan J. M. Halls, ${ }^{C}$ Martin Heeney ${ }^{\star a}$ and John C. de Mello*a

Accepted 2nd October 2013

DOI: $10.1039 / c 3 m h 00066 d$

rsc.li/materials-horizons

We report the highly controlled synthesis of conjugated random copolymers in a droplet-based microfluidic reactor. Using two optically distinct polymers, poly(3-hexylthiophene) (P3HT) and poly(3-hexylselenophene) (P3HS), a series of highly regioregular random copolymers is generated with physical properties intermediate to those of the parent homopolymers. Analysis by ${ }^{1} \mathrm{H}$ nuclear magnetic resonance spectroscopy reveals the co-polymerisation process to follow ideal Bernoullian behavior.

Semiconducting polymers are attracting widespread interest due to their potential applications in lighting, displays and solar cells, and for their compatibility with high volume manufacturing on flexible plastic substrates. ${ }^{1-4}$ However, with the latest generations of semiconducting polymers now nearing application-viable levels of performance, ${ }^{1}$ an urgent need has arisen for new quality-assured production methods that can scale to industrially relevant quantities, while providing reliable batch-to-batch reproducibility in terms of stoichoimetry, molecular weight and structural order.

We recently described a new strategy for the scalable synthesis of high quality semiconducting polymers based on the use of droplet microfluidics. ${ }^{5}$ In brief, by injecting monomer feedstock into a fast-flowing stream of immiscible carrier fluid, a series of near-identical sub $\mu \mathrm{L}$ droplets is generated inside the carrier fluid, each of which acts as a self-contained microscale reaction vessel. The small droplet size ensures rapid equilibration of composition and temperature, and so provides a highly uniform environment for polymerisation. Importantly, since materials throughput can be raised independently of droplet

${ }^{a}$ Centre for Plastic Electronics, Department of Chemistry, Imperial College London, Exhibition Road, South Kensington, London SW7 2AY, UK. E-mail: m.heeney@ imperial.ac.uk; j.demello@imperial.ac.uk

${ }^{b}$ Department of Chemistry, Texas A\&M University at Qatar, P.O. Box 23874, Doha, Qatar

${ }^{c}$ Solar Press, 2 Royal College Street, London NW1 ONH, UK

$\dagger$ Electronic supplementary information (ESI) available. See DOI: $10.1039 / \mathrm{c} 3 \mathrm{mh} 00066 \mathrm{~d}$ volume (by ramping up the rate of droplet generation while keeping the droplet size fixed), production levels can in principle be scaled-up indefinitely without detriment to yield or product quality. To validate the droplet approach we previously used the Grignard metathesis (GRIM) route to synthesise device-grade poly(3-hexylthiophene) (P3HT) with number average molecular weights up to $50 \mathrm{kDa}$. The materials had high regioregularities ( $\mathrm{RR}>97 \%$ ) and low polydispersity indices (PDI < 1.7), with production rates of up to $60 \mathrm{~g}$ per day being readily achievable without leakage or fouling. Importantly the droplet approach was found to facilitate the synthesis of high molecular weight polymers, which are challenging to synthesise in conventional single-phase flow reactors where precipitation of product on the reactor walls can lead to blockage.

Here we report the extension of the droplet method to the preparation of statistical copolymers. Using P3HT and poly(3hexylselenophene) (P3HS) as parent materials, we demonstrate the controlled synthesis of a series of statistical copolymers, varying in composition from $100 \%$ P3HT to $100 \%$ P3HS. P3HT is amongst the most widely studied of conjugated polymers due to its formation of semicrystalline thin-films with good hole-transporting properties and broad spectral coverage up to $600 \mathrm{~nm}$ - favourable properties for thin-film transistors and organic photovoltaics. ${ }^{6}$ P3HS, the selenium analogue of P3HT, has comparable hole transporting properties but due to its deeper-lying LUMO level has improved spectral coverage, extending to $760 \mathrm{~nm} .^{7,8}$ Copolymerisation of 3-hexylthiophene and 3-hexylselenophene has previously been found to yield materials with absorption spectra intermediate to those of the two parent polymers, and was selected here as an attractive test reaction for evaluating the levels of synthetic control achievable using droplet-based synthesis., ${ }^{9,10}$

All materials were prepared here via the Grignard metathesis route of McCullough et al., ${ }^{11}$ which provides a simple method for preparing highly regioregular polymers from readily prepared dibrominated monomers (see Scheme 1). Reaction of the monomers with one equivalence of an alkyl Grignard reagent - typically isopropylmagnesium chloride (iPrMgCl) or 


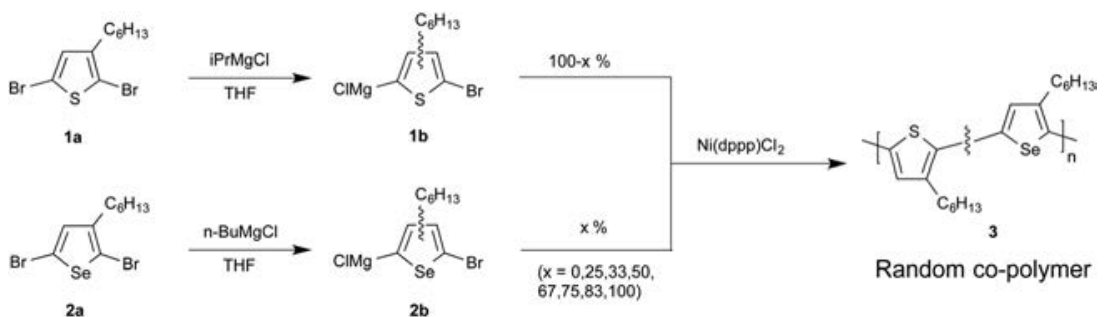

Scheme 1 Synthesis of poly(3-hexylthiophene)-co-poly(3-hexylselenophene) [P3HT-co-P3HS] (3).

$n$-butylmagnesium chloride $(n$-BuMgCl $)$ - results in a metathesis reaction that affords the more stable thienyl/selenyl Grignard. For asymmetric monomers such as 2,5-dibromo-3hexylthiophene and 2,5-dibromo-3-hexylselenophene, formation of the Grignard at the 5-position is favoured over formation at the 2-position by an approximate ratio of four to one. ${ }^{7,11}$ Polymerisation is readily initiated by the addition of a Kumadatype nickel or palladium catalyst, with 1,3-bis[diphenylphosphinopropane]nickel(II) chloride $\left[\mathrm{Ni}(\mathrm{dppp}) \mathrm{Cl}_{2}\right]$ being the catalyst of choice due to the high regioregularity of the resultant material. $\$$ The chain-growth character ${ }^{\mathbf{1 2 , 1 3}}$ of the polymerization yields polymers with well controlled, narrow weight distributions. ${ }^{\mathbf{1 4 - 1 8}}$

In adapting the GRIM method to the droplet synthesis of P3HT, we previously dispersed the $\mathrm{Ni}(\mathrm{dppp}) \mathrm{Cl}_{2}$ catalyst in the carrier fluid since direct dissolution in THF under $\mathrm{N}_{2}$ was found to cause ligand detachment, leading to bleaching of its red coloration and a severe loss of catalytic activity. One drawback of dispersing the catalyst in the carrier fluid is the difficulty of controlling the catalyst-to-monomer ratio since the catalyst enters the reagent phase diffusively via the droplet surface. In the current work, the catalyst was dissolved directly in THF, with the problem of catalyst deactivation being solved by codissolving excess dppp ligand alongside the $\mathrm{Ni}(\mathrm{dppp}) \mathrm{Cl}_{2}$. (A $1: 1$ weight ratio of ligand to catalyst was found to be sufficient).
Dissolution of the catalyst in the reagent phase provides a more versatile and rigorously defined chemical environment for the polymerisation since the ratio of catalyst to monomer can be precisely controlled by fixing their relative rates of infusion.

The catalyst solution was prepared by dissolving $10 \mathrm{mg}$ $\mathrm{Ni}(\mathrm{dppp}) \mathrm{Cl}_{2}(18.44 \mu \mathrm{mol})$ and $10 \mathrm{mg}$ dppp $(24.25 \mu \mathrm{mol}, 1.31$ equiv.) in $10 \mathrm{~mL}$ anhydrous THF (a-THF). The catalyst solution and $0.25 \mathrm{M}$ solutions of monomers $\mathbf{1 b}$ and $\mathbf{2 b}$ in a-THF were loaded into three separate gas-tight syringes, each of which was then mounted on an individual syringe pump. (See ESI $\dagger$ for a description of the synthesis routes for the monomers.) The two monomer solutions were injected from their respective syringes into a static 1.7 $\mu \mathrm{L}$ Y-shaped mixer, Fig. 1a. The selenophene/ thiophene mixture from the mixer and the catalyst/ligand solution from the third syringe were fed into a three-input droplet generator (see Fig. 1b and d), using Galden HT-170 perfluorinated polyether (PFPE) (Solvay Solexis) supplied by a fourth syringe pump as an immiscible carrier fluid. The outlet of the droplet generator was connected to a $2.25 \mathrm{~m}$ length of polytetrafluoroethylene (PTFE) tubing (1 mm ID, $2 \mathrm{~mm}$ OD, Bohlender). The innermost $2 \mathrm{~m}(1.57 \mathrm{~mL})$ of the PTFE tubing was held in a flat spiral arrangement using a 3D-printed polylactic acid plastic framework and fully immersed in paraffin oil at $55{ }^{\circ} \mathrm{C}$, Fig. 1c and SI4. $\dagger$ The effluent was collected in methanol-filled vials for quenching and precipitation of the



Fig. 1 Schematic of droplet reactor, comprising: (a) a static mixer for controlling the selenophene to thiophene ratio; (b) a three-input droplet generator into which the carrier fluid, catalyst solution and premixed monomer solution are injected; and (c) coiled PTFE tubing of inner diameter $1 \mathrm{~mm}$ in a temperature-stabilised oil-bath. The stated flow conditions correspond to a residence time of $436 \mathrm{~s}$ in the oil-bath and a catalyst loading of $0.74 \mathrm{~mol} \%$ relative to the monomer. (d) A schematic of the droplet generator. 
product. To ensure a constant heating time of $436 \mathrm{~s}$ for all

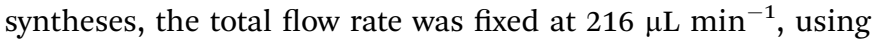
flow-rates of 108,54 and $54 \mu \mathrm{L} \min ^{-1}$ for the carrier, catalyst, and combined $(\mathbf{1 b}+\mathbf{2 b})$ monomer streams respectively. The composition of the reaction mixture was varied by tuning the ratio of the selenophene and thiophene infusion rates, while holding their combined value constant at $54 \mu \mathrm{L} \mathrm{min}{ }^{-1}$. Under these flow conditions, the catalyst loading was fixed at $0.74 \mathrm{~mol}$ $\%$ relative to the total monomer concentration. (The complete reactor setup and droplet generation process is described in the ESI $\dagger$.)

Eight different pairings of monomer flow-rates were used to prepare the series of copolymers, ranging from $100 \%$ thiophene to $100 \%$ selenophene. (In the case of the two homopolymers, the Y-mixer was removed and the relevant monomer was injected directly into the PTFE reaction channel from its syringe.) After each change of flow rate, the system was allowed to settle for eight minutes before commencing sample collection. Each sample was collected in a separate methanol-filled vial over a four minute period. Refractive index size-exclusion chromatography (calibrated to polystyrene standards) showed all copolymers to have broadly similar number average molecular weights in the range $36 \mathrm{kDa}$ to $44 \mathrm{kDa}$, with relatively low polydispersity indices in the range 1.3-1.5 (see Fig. SI and Table SI $\dagger \dagger$ ).

${ }^{1} \mathrm{H}$ NMR was used to determine the absolute ratio of selenophene to thiophene in the product, the statistical nature of the copolymerization process, and the structural regioregularity. The chemical environment of the ring proton is affected by the heteroatom in the ring and the heterocycle coupled at the 5 -position. Consequently, as seen in the left hand spectra of Fig. 2a, for fully regioregular materials there are four distinct couplings evident in the NMR: Se-S, S-S, Se-Se and S-Se at 6.95, 7.01, 7.16 and $7.22 \mathrm{ppm}$, respectively. (Note, the unit containing the ring proton is specified here in bold, and signals were corrected for interference by the $\mathrm{CHCl}_{3}$ signal.) The relative frequencies of the four coupling types can be straightforwardly determined from the relative integrals of the coupling peaks.

For a fully random copolymerization, the two monomers are added to the polymer chain with equal probabilities at all stages of the polymerisation. This results in the formation of a Bernoullian copolymer in which the relative frequency of each coupling type is determined by the product of the mole fractions of the corresponding monomers in the initial reaction mixture. ${ }^{19}$ Fig. 2b-e show for the four coupling types, the experimentally determined coupling frequencies versus the mole fraction of selenophene in the initial reaction mixture. Also shown in Fig. $2 \mathrm{f}$ is the mole fraction of selenophene in the final copolymer versus the mole fraction of selenophene in the initial reaction mixture. All experimental data (blue markers) adhered closely to ideal Bernoullian behavior ${ }^{19}$ (red curves), confirming the random nature of the copolymerisation process. The near-ideal nature of the coupling data demonstrates the very high levels of reaction control that can be attained using the droplet approach, with the stoichiometry of the final copolymers being quantitatively determined in all cases by the relative infusion rates of the two monomer solutions.
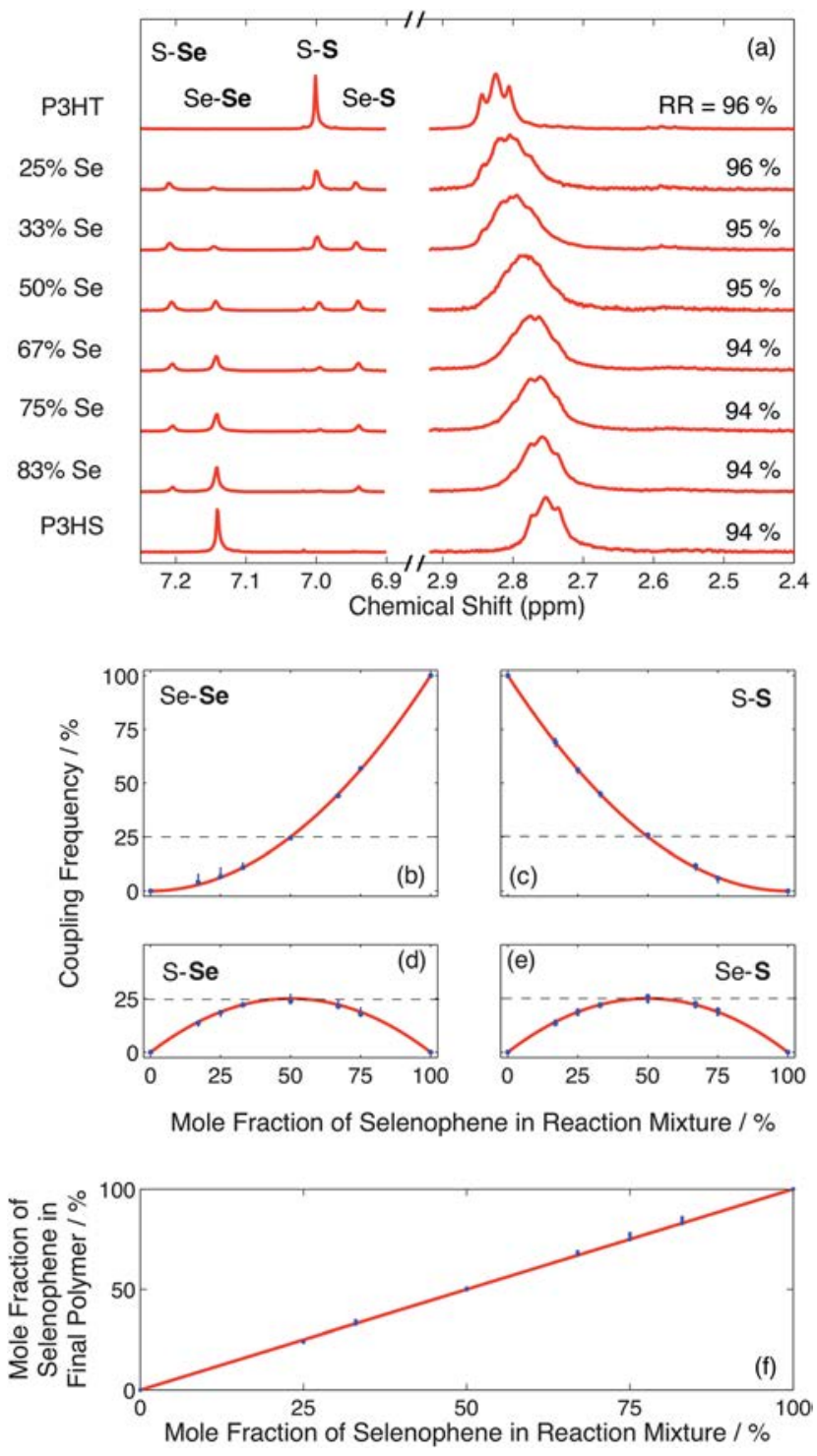

Fig. 2 (a) ${ }^{1} \mathrm{H}$ NMR spectra showing the ring proton peaks (left) and $\alpha$ methylene proton peaks (right) of a series of droplet-synthesised P3HT-CO-P3HS statistical copolymers, ranging in composition from $100 \%$ P3HT to $100 \%$ P3HS; the $y$-axis labels denote the mole fraction of selenophene in the initial reaction mixture. (b-e) Relative frequencies of the four coupling types versus mole fraction of selenophene in the initial reaction mixture. (f) Mole fraction of selenophene in the final polymer versus mole fraction of selenophene in the initial reaction mixture. The relative coupling frequencies and selenophene content were determined from the areas under the ring proton peaks in (a), correcting the area under the selenophene peak for interference by $\mathrm{CHCl}_{3}$. The blue markers in (b) to (f) indicate experimental data (with the vertical lines denoting extreme error bounds), while the red curves indicate ideal Bernoullian behavior.

As shown in the right hand spectra of Fig. 2a the two homopolymers exhibited clearly resolved head-to-tail $\alpha$-methylene peaks, corresponding to splitting by the neighbouring aliphatic protons. The copolymers exhibited peaks from both parent polymers, albeit with relatively poor resolution between individual peaks due to overlapping contributions from the heterocouplings. In the case of the 50 : 50 copolymer, a single 
broad peak was observed with no discernible fine structure. A steady shift in the peak position of the head-to-tail $\alpha$-methylene proton from 2.83 to $2.76 \mathrm{ppm}$ was observed in moving through the series of copolymers from pure P3HT to pure P3HS, consistent with the random nature of the copolymerisation. Integration of the head-to-tail and head-to-head/tail-to-tail $\alpha$-methylene signals ${ }^{20}$ indicated only a slight change in regioregularity from $96 \%$ to $94 \%$ in moving from pure $\mathrm{P} 3 \mathrm{HT}$ to pure P3HS, indicating a strong preference for head-to-tail coupling in all cases.

Fig. 3a shows normalised absorption spectra in chlorobenzene for the eight samples, with each sample showing a single absorption peak that progressively red-shifted and slightly broadened as the mole fraction of selenophene in the initial reaction mixture was raised from $0 \%$ to $100 \%$. Plotting the peak wavelength versus selenophene content (Fig. 3b) yielded a linear trend from $455 \mathrm{~nm}$ for P3HT to $498 \mathrm{~nm}$ for P3HS. The progressive shift in spectra and absence of multiple absorption features are consistent with the formation of statistical as opposed to block copolymers. We note that the ability to finely tune the optical properties of the copolymers through simple adjustments to the monomer infusion rates provides a simple and effective means of optimising the copolymer characteristics for a desired application.

In conclusion we have extended the droplet method to the synthesis of random copolymers of poly(3-hexylthiophene) and poly(3-hexylselenophene) to produce a series of statistical
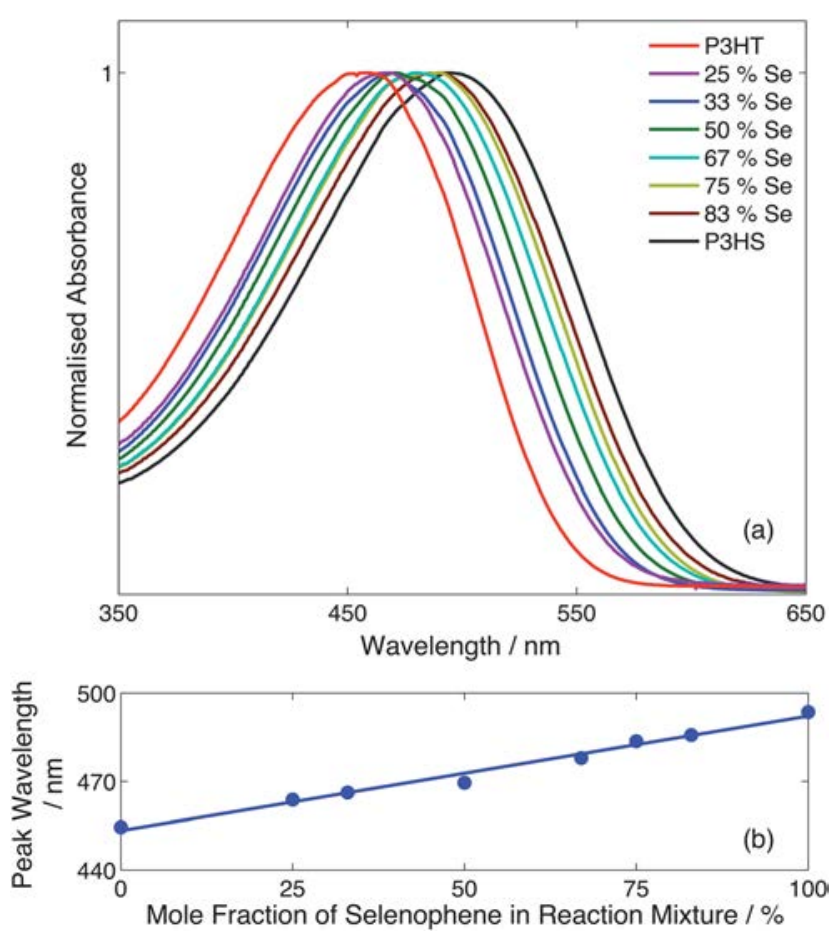

Fig. 3 (a) Solution-phase absorption spectra in chlorobenzene for the series of droplet-synthesised P3HT-CO-P3HS random copolymers. The legend denotes the mole fraction of selenophene in the initial reaction mixture. (b) Peak absorption wavelength versus mole fraction of selenophene in the initial reaction mixture; the solid line is a guide to the eye. copolymers, ranging in composition from $100 \%$ P3HT to $100 \%$ P3HS. The ring proton signals in the ${ }^{1} \mathrm{H}$ NMR indicated a fully random (Bernoullian) copolymerisation in which the two monomers are incorporated into the chain with equal probabilities at all stages of chain growth, resulting in a final stoichiometry that matches the initial composition of the reaction mixture. Analysis of the $\alpha$-methylene proton region indicated that all copolymers had a strong preference for head-to-tail coupling, with the regioregularity shifting only slightly from 96 to $94 \%$ as the selenophene content was increased. The copolymers exhibited composition-dependent absorption spectra intermediate to those of the parent polymers, with the optical band-gap varying linearly from 455 to $498 \mathrm{~nm}$ as the mole fraction of selenophene in the feedstock was increased from $0 \%$ to $100 \%$. The high degree of reaction control demonstrated here, combined with the excellent stability and ready scalability reported previously, confirms droplet synthesis to be a highly effective method for the controlled production of conjugated polymers with significant advantages over conventional batch methods.

\section{Acknowledgements}

JB is funded under an EPSRC Doctoral Training Centre in Plastic Electronics (grant number EP/G037515/1) and holds an Industrial Fellowship with the Royal Commission for the Exhibition of 1851. SK acknowledges financial support from the EPSRC under its Knowledge Transfer Secondment scheme and from the Technology Strategy Board (Grant name CAMPFiRe). JdM acknowledges financial support under the Royal Society Industry Fellowship scheme. The authors acknowledge additional financial support from the EPSRC under grant number EP/G031088/1.

\section{Notes and references}

$\ddagger$ The high regioregularity derives from the slow reaction kinetics of the (minority) monomers having the Grignard at the 2 position, which minimises their participation in the polymerisation and so ensures predominantly head-to-tail couplings by the majority monomer. ${ }^{16}$

1 A. J. Heeger, Chem. Soc. Rev., 2010, 39, 2354-2371.

2 G. Li, R. Zhu and Y. Yang, Nat. Photonics, 2012, 6, 153-161.

3 R. Parashkov, E. Becker, T. Riedl, H.-H. Johannes and W. Kowalsky, Proc. IEEE, 2005, 93, 1321-1329.

4 F. C. Krebs, Sol. Energy Mater. Sol. Cells, 2009, 93, 394-412. 5 J. H. Bannock, S. H. Krishnadasan, A. M. Nightingale, C. P. Yau, K. Khaw, D. Burkitt, J. J. M. Halls, M. Heeney and J. C. de Mello, Adv. Funct. Mater., 2013, 23, 2123-2129.

6 A. Marrocchi, D. Lanari, A. Facchetti and L. Vaccaro, Energy Environ. Sci., 2012, 5, 8457.

7 M. Heeney, W. Zhang, D. J. Crouch, M. L. Chabinyc, S. Gordeyev, R. Hamilton, S. J. Higgins, I. McCulloch, P. J. Skabara, D. Sparrowe and S. Tierney, Chem. Commun., 2007, 5061-5063.

8 A. M. Ballantyne, L. Chen, J. Nelson, D. D. C. Bradley, Y. Astuti, A. Maurano, C. G. Shuttle, J. R. Durrant, M. Heeney, W. Duffy and I. McCulloch, Adv. Mater., 2007, 19, 4544-4547. 
9 J. Hollinger, A. A. Jahnke, N. Coombs and D. S. Seferos, J. Am. Chem. Soc., 2010, 132, 8546-8547.

10 E. F. Palermo and A. J. McNeil, Macromolecules, 2012, 45, 5948-5955.

11 R. Loewe, S. Khersonsky and R. D. McCullough, Adv. Mater., 1999, 11, 250-253.

12 A. Yokoyama, R. Miyakoshi and T. Yokozawa, Macromolecules, 2004, 37, 1169-1171.

13 E. E. Sheina, J. Liu, M. C. Iovu, D. W. Laird and R. D. McCullough, Macromolecules, 2004, 37, 3526-3528.

14 R. Miyakoshi, A. Yokoyama and T. Yokozawa, J. Am. Chem. Soc., 2005, 127, 17542-17547.
15 M. C. Stefan, M. P. Bhatt, P. Sista and H. D. Magurudeniya, Polym. Chem., 2012, 3, 1693-1701.

16 R. S. Loewe, P. C. Ewbank, J. Liu, L. Zhai and R. D. McCullough, Macromolecules, 2001, 34, 4324-4333.

17 K. Okamoto and C. K. Luscombe, Polym. Chem., 2011, 2, 2424.

18 A. Kiriy, V. Senkovskyy and M. Sommer, Macromol. Rapid Commun., 2011, 32, 1503-1517.

19 G. Odian, Principles of Polymerization, Wiley-Interscience, New York, 4th edn, 2004.

20 T. A. Chen, X. Wu and R. D. Rieke, J. Am. Chem. Soc., 1995, 117, 233-244. 